\title{
An underlying symmetry determines all elements of CKM and PMNS up to a universal constant?
}

\author{
Hong-Wei $\mathrm{Ke}^{1 *}$ and Xue-Qian $\mathrm{Li}^{2 \dagger}$ \\ 1 School of Science, Tianjin University, Tianjin 300072, China \\ 2 School of Physics, Nankai University, Tianjin 300071, China
}

\begin{abstract}
Observing the CKM matrix elements written in different parametrization schemes, one can notice obvious relations among the sine-values of the $\mathrm{CP}$ phases in those schemes. Using the relations, we establish a few parametrization-independent equations, by which the matrix elements of the CKM matrix can be completely fixed up to a universal parameter. If it is true, we expect that there should exist a hidden symmetry in the nature which determines the relations. Moreover, it requires a universal parameter, naturally it would be the famous Jarlskog invariant which is also parametrization independent. Thus the four parameters (three mixing angles and one CP phase) of the CKM matrix are not free, but determined by the symmetry and the universal parameter. As we generalize the rules to the PMNS matrix for neutrino mixing, the CP phase of the lepton sector is predicted to be within a range of $0 \sim 59^{\circ}$ centered at $39^{\circ}$ (in the $\mathrm{P}_{a}$ parametrization) which will be tested in the future experiments.
\end{abstract}

PACS numbers: 12.15.Ff, 14.60.Pq, 12.15.Hh

\footnotetext{
*khw020056@hotmail.com.

$\dagger$ lixq@nankai.edu.cn
} 


\section{INTRODUCTION}

Since Cabibbo first noted the difference between the decays of neutron $\left(n \rightarrow p+e^{-}+\bar{\nu}_{e}\right)$ and muon $\left(\mu \rightarrow e+\bar{\nu}_{e}+\nu_{\mu}\right)$ and suggested there is a mixing angle between $\mathrm{d}$ and s quarks[1], and the mixing perfectly explained the data of the $\beta$-decays of $\Sigma^{-}$and $\Lambda$ [2]. Later the CKM matrix [3] has been proposed to mix the three generation quarks. In the $3 \times 3$ matrix there are three mixing angles and a CP phase which seem to be completely independent of each other. The mixing is understood as that the eigen-basis of weak interaction is not the same as that of mass, so matching them, a unitary transformation matrix must be introduced [4 6]. From then on, the research field about quark mixing has been thoroughly investigated and exploration of its source has never ceased. In analog to the quark sector, the PontecorvoMaki-Nakawaga-Sakata (PMNS) matrix [7, 8] relates the lepton flavor eigenstates with the mass eigenstates. Thus it is natural to consider that there might be an underlying symmetry which results in the practical CKM and PMNS matrices after symmetry breaking. Recent studies on these matrices indicate that there exist the quark-lepton complementarity and self-complementarity [9 15] which hint an existence of a higher symmetry. All the progress in this area inspires a trend of searching for whether such a hidden symmetry indeed exists and moreover investigation of its phenomenological implication is also needed.

The key point is to investigate whether there exist some relations among the matrix elements of the CKM and/or PMNS matrices which seem to be completely independent if there is no such a symmetry to make an arrangement. There are nine parametrization schemes which manifest the mixing in different ways. Therefore, we expect that some relations among the parameters of these nine schemes might hint a hidden symmetry if they indeed exist. Listing the parameters (the mixing angles and CP phase) of the nine schemes in a table and staring on them, we notice that there are relations among the sine values of the CP phases in these schemes. Namely, for the nine parametrization schemes, we find equalities among those $\sin \delta_{n}$ i.e. $\sin \delta_{a} \approx \sin \delta_{e}, \sin \delta_{b} \approx \sin \delta_{c}, \sin \delta_{d} \approx \sin \delta_{e}, \sin \delta_{f} \approx \sin \delta_{h}$ and $\sin \delta_{h} \approx \sin \delta_{i}$ where the the subscripts $a$ through $i$ refer to the nine parametrization schemes. Considering the relations not to be accidental, we would be tempted to believe there is a hidden symmetry. Then associating the expressions of Jarlskog invariant which include sine values of the CP phases in all schemes with the experimentally measurable CKM matrix elements $\left|U_{j k}\right|$ and using the above relations, we establish several equalities which do not depend on any concrete parametrization scheme at all. In principle when we apply the equalities to a special scheme, the solutions of those equalities would give the values of the parameters of the concerned scheme up to a universal dimensionless constant.

What is the universal dimensionless constant? Surely it must be a scheme-independent quantity and has clear physics significance. Naturally, one can conjecture that the Jarlskog invariant [16] which is related to the $\mathrm{CP}$ violation of hadrons is the best choice. Thus we would accept the allegation.

However, we also notice that all the equalities only approximately hold, even though the coincidence is very high. This can be well understood that the hidden symmetry is slightly broken, but some characteristics of the original symmetry is partly retained after 
the breaking. The approximate equalities are listed in the context of this work.

We generalize the relations among the CKM matrix to the PMNS case and find that all the aforementioned equalities also hold for the lepton sector, even though the accuracy is not as high as for the quark sector. Along the line, we further investigate the induced phenomenological implication which may be tested in more accurate neutrino experiments.

The paper is organized as follows. After the introduction we present those relations in section II. In section III, we check these relations numerically. In section IV we will discuss the implications about the possible hidden symmetry and draw our conclusion.

\section{RELATIONS AMONG ELEMENTS OF THE CKM AND PMNS MATRICES}

In this section we show how to obtain the relations among the elements of the CKM and as well the PMNS matrices.

\section{A. The mixing of fermions in standard model}

Mixing among different flavors of quarks (leptons) via the CKM (PMNS) matrix has been firmly recognized and widely applied to phenomenological studies of hadronic processes. The Lagrangian of the weak interaction reads

$$
\mathcal{L}=\frac{g}{\sqrt{2}} \bar{U}_{L} \gamma^{\mu} V_{C K M} D_{L} W_{\mu}^{+}+\frac{g}{\sqrt{2}} \bar{E}_{L} \gamma^{\mu} V_{P M N S} N_{L} W_{\mu}^{+}+\text {h.c. },
$$

where $U_{L}=\left(u_{L}, c_{L}, t_{L}\right)^{T}, D_{L}=\left(d_{L}, s_{L}, b_{L}\right)^{T}, E_{L}=\left(e_{L}, \mu_{L}, \tau_{L}\right)^{T}$ and $N_{L}=\left(\nu_{1}, \nu_{2}, \nu_{3}\right)^{T}$. $V_{C K M}$ and $V_{P M N S}$ are the CKM and PMNS matrices respectively. The $3 \times 3$ mixing matrices are written as

$$
V=\left(\begin{array}{lll}
U_{11} & U_{12} & U_{13} \\
U_{21} & U_{22} & U_{23} \\
U_{31} & U_{32} & U_{33}
\end{array}\right)
$$

Generally, for a $3 \times 3$ unitary matrix there are four independent parameters, namely three mixing angles and one CP-phase. There can be various schemes to parameterize the matrix and in literature, nine different schemes are presented and widely applied. They are clearly listed in Ref.[14]. Here we try to collect those elements for various parametrization schemes in Tab. П where $\theta_{n j}$ and $\delta_{n}$ are the mixing angles and CP-phase. For clarity, we use $\vartheta_{n j}$ and $\delta_{n}^{\prime}$ to denote the corresponding quantities in the PMNS matrix.

The $\mathrm{P}_{a}$ parametrization i.e. $\mathrm{P} 1$ parametrization in[14], can be realized via a serial rotations

$$
V=R_{23}\left(\theta_{a 2}\right) R_{31}\left(\theta_{a 3}, \delta_{a}\right) R_{12}\left(\theta_{a 1}\right),
$$

and the relevant rotation matrices $R_{23}, R_{31}, R_{12}$ were given in Ref.[14].

Then for the $\mathrm{P}_{a}$ parametrization an explicit expression is shown as

$$
V=\left(\begin{array}{ccc}
c_{a 1} c_{a 3} & s_{a 1} c_{a 3} & s_{a 3} \\
-c_{a 1} s_{a 2} s_{a 3}-s_{a 1} c_{a 2} e^{i \delta_{a}} & -s_{a 1} s_{a 2} s_{a 3}+c_{a 1} c_{a 2} e^{i \delta_{a}} & s_{a 2} c_{a 3} \\
-c_{a 1} s_{a 2} s_{a 3}+s_{a 1} s_{a 2} e^{i \delta_{a}} & -s_{a 1} s_{a 2} s_{a 3}-c_{a 1} s_{a 2} e^{i \delta_{a}} & c_{a 2} c_{a 3}
\end{array}\right) .
$$


TABLE I: Nine different schemes for CKM matrix

\begin{tabular}{|c|c|c|}
\hline Scheme & Jarlskog invariant & Mixing angles and CP-phase \\
\hline $\mathrm{P}_{a}: R_{23}\left(\theta_{a 2}\right) R_{31}\left(\theta_{a 3}, \delta_{a}\right) R_{12}\left(\theta_{a 1}\right)$ & $J_{a}=s_{a 1} s_{a 2} s_{a 3} c_{a 1} c_{a 2} c_{a 3}^{2} \sin \delta_{a}$ & $\begin{array}{c}\theta_{a 1}=\arcsin \frac{\left|U_{12}\right|}{\left|U_{11}\right|}, \theta_{a 2}=\arctan \frac{U_{23} \mid}{\left|U_{33}\right|} \\
\theta_{a 3}=\arcsin \left|U_{13}\right|, \delta_{a}=\left(69.10_{-3.85}^{+2.02}\right)^{\circ}\end{array}$ \\
\hline $\mathrm{P}_{b}: R_{12}\left(\theta_{b 3}\right) R_{23}\left(\theta_{b 2}, \delta_{b}\right) R_{12}^{-1}\left(\theta_{b 1}\right)$ & $J_{b}=s_{b 1} s_{b 2}^{2} s_{b 3} c_{b 1} c_{b 2} c_{b 3} \sin \delta_{b}$ & $\begin{array}{c}\theta_{b 1}=\arctan \frac{\left|U_{31}\right|}{\left|U_{32}\right|}, \theta_{b 2}=\arccos \left|U_{33}\right| \\
\theta_{b 3}=\arctan \frac{\left|U_{13}\right|}{\left|U_{23}\right|}, \delta_{b}=\left(89.69_{-3.95}^{+2.29}\right)^{\circ}\end{array}$ \\
\hline $\mathrm{P}_{c}: R_{23}\left(\theta_{c 2}\right) R_{12}\left(\theta_{c 1}, \delta_{c}\right) R_{23}^{-1}\left(\theta_{c 3}\right)$ & $J_{c}=s_{c 1}^{2} s_{c 2} s_{c 3} c_{c 1} c_{c 2} c_{c 3} \sin \delta_{c}$ & $\begin{array}{c}\theta_{c 1}=\arccos \left|U_{11}\right|, \theta_{c 2}=\arctan \frac{\left|U_{31}\right|}{\left|U_{21}\right|} \\
\theta_{c 3}=\arctan \frac{\left|U_{13}\right|}{\left|U_{12}\right|}, \delta_{c}=\left(89.29_{-2.33}^{+3.99}\right)^{\circ}\end{array}$ \\
\hline $\mathrm{P}_{d}: R_{23}\left(\theta_{d 2}\right) R_{12}\left(\theta_{d 1}, \delta_{d}\right) R_{31}^{-1}\left(\theta_{3 d}\right)$ & $J_{d}=s_{d 1} s_{d 2} s_{d 3} c_{d 1}^{2} c_{d 2} c_{d 3} \sin \delta_{d}$ & $\begin{array}{c}\theta_{d 1}=\arcsin \left|U_{12}\right|, \theta_{d 2}=\arctan \frac{\left|U_{32}\right|}{\left|U_{22}\right|} \\
\theta_{d 3}=\arctan \frac{\left|U_{13}\right|}{\left|U_{11}\right|}, \delta_{d}=\left(111.95_{-2.02}^{+3.82}\right)^{\circ}\end{array}$ \\
\hline $\mathrm{P}_{e}: R_{31}\left(\theta_{e 3}\right) R_{23}\left(\theta_{e 2}, \delta_{e}\right) R_{12}^{-1}\left(\theta_{e 1}\right)$ & $J_{e}=s_{e 1} s_{e 2} s_{e 3} c_{e 1} c_{e 2}^{2} c_{e 3} \sin \delta_{e}$ & $\begin{array}{c}\theta_{e 1}=\arctan \frac{\left|U_{21}\right|}{\left|U_{22}\right|}, \theta_{e 2}=\arcsin \left|U_{23}\right| \\
\theta_{e 3}=\arctan \frac{\left|U_{13}\right|}{\left|U_{33}\right|}, \delta_{e}=\left(110.94_{-2.02}^{+3.85}\right)^{\circ}\end{array}$ \\
\hline $\mathrm{P}_{f}: R_{12}\left(\theta_{f 1}\right) R_{31}\left(\theta_{f 3}, \delta_{f}\right) R_{23}^{-1}\left(\theta_{f 2}\right)$ & $J_{f}=s_{f 1} s_{f 2} s_{f 3} c_{f 1} c_{f 2} c_{f 3}^{2} \sin \delta_{f}$ & $\begin{array}{c}\theta_{f 1}=\arctan \frac{\left|U_{21}\right|}{\left|U_{11}\right|}, \theta_{f 2}=\arctan \frac{\left|U_{32}\right|}{\left|U_{33}\right|} \\
\theta_{f 3}=\arcsin \left|U_{31}\right|, \delta_{f}=\left(22.72_{-1.18}^{+1.25}\right)^{\circ}\end{array}$ \\
\hline $\mathrm{P}_{g}: R_{31}\left(\theta_{g 3}\right) R_{12}\left(\theta_{g 1}, \delta_{g}\right) R_{31}^{-1}\left(\theta_{g 2}\right)$ & $J_{g}=s_{g 1}^{2} s_{g 2} s_{g 3} c_{g 1} c_{g 2} c_{g 3} \sin \delta_{9}$ & $\begin{array}{l}\theta_{g 1}=\arccos \left|U_{22}\right|, \theta_{g 2}=\arctan \frac{\left|U_{23}\right|}{\left|U_{21}\right|} \\
\theta_{g 3}=\arctan \frac{\left|U_{32}\right|}{\left|U_{12}\right|}, \delta_{g}=\left(1.08_{-0.06}^{+0.06}\right)^{\circ}\end{array}$ \\
\hline $\mathrm{P}_{h}: R_{12}\left(\theta_{h 1}\right) R_{23}\left(\theta_{h 2}, \delta_{h}\right) R_{31}^{-1}\left(\theta_{h 3}\right)$ & $J_{h}=s_{h 1} s_{h 2} s_{h 3} c_{h 1} c_{h 2}^{2} c_{h 3} \sin \delta_{8}$ & $\begin{array}{c}\theta_{h 1}=\arctan \frac{\left|U_{12}\right|}{\left|U_{22}\right|}, \theta_{h 2}=\arcsin \left|U_{32}\right| \\
\theta_{h 3}=\arctan \frac{\left|U_{31}\right|}{\left|U_{33}\right|}, \delta_{h}=\left(157.31_{-1.25}^{+1.18}\right)^{\circ}\end{array}$ \\
\hline $\mathrm{P}_{i}: R_{31}\left(\theta_{i 3}\right) R_{12}\left(\theta_{i 1}, \delta_{i}\right) R_{23}^{-1}\left(\theta_{i 2}\right)$ & $J_{i}=s_{i 1} s_{i 2} s_{i 3} c_{i 1}^{2} c_{i 2} c_{i 3} \sin \delta_{i}$ & $\begin{array}{c}\theta_{i 1}=\arcsin \left|U_{21}\right|, \theta_{i 2}=\arctan \frac{\left|U_{23}\right|}{\left|U_{22}\right|} \\
\theta_{i 3}=\arctan \frac{\left|U_{31}\right|}{\left|U_{11}\right|}, \delta_{i}=\left(158.32_{-1.20}^{+1.13}\right)^{\circ}\end{array}$ \\
\hline
\end{tabular}

Here $s_{a j}$ and $c_{a j}$ denote $\sin \theta_{a j}$ and $\cos \theta_{a j}$ with $j=1,2,3$. The corresponding expressions in other schemes $\mathrm{P}_{n}$ can be obtained in similar ways.

Thanks to hard experimental measurements on the weak hadronic transitions where the CKM matrix is involved, the mixing parameters for the quark sector are [14]

$$
\theta_{a 1}=\left(13.023_{-0.038}^{+0.038}\right)^{\circ}, \theta_{a 2}=\left(2.360_{-0.038}^{+0.065}\right)^{\circ}, \theta_{a 3}=\left(0.201_{-0.008}^{+0.010}\right)^{\circ}, \delta_{a}=\left(69.10_{-3.85}^{+2.02}\right)^{\circ} .
$$

Similarly, the parameters in the PMNS matrix [14] are

$$
\vartheta_{a 1}=\left(33.65_{-1.00}^{+1.11}\right)^{\circ}, \vartheta_{a 2}=\left(38.41_{-1.21}^{+1.40}\right)^{\circ}, \vartheta_{a 3}=\left(8.93_{-0.48}^{+0.46}\right)^{\circ},
$$

which are directly measured by the neutrino-involved experiments, especially the neutrino oscillations, but so far the CP-phase $\delta_{a}^{\prime}$ in the lepton sector is undetermined yet.

\section{B. Several relations for CKM}

A close observation on the values of $\delta_{n}$ in different schemes shows several approximate equalities

$$
\sin \delta_{a} \approx \sin \delta_{e}, \sin \delta_{b} \approx \sin \delta_{c}, \sin \delta_{d} \approx \sin \delta_{e}, \sin \delta_{f} \approx \sin \delta_{h}, \sin \delta_{h} \approx \sin \delta_{i} .
$$


It is well known that the Jarlskog invariant is independent of different schemes, so using the above relations in Eq.(7) and replacing the $s_{n j}$ and $c_{n j}$ with the ratios of modules of corresponding elements in the expressions of Jarlskog invarian, one can deduce several interesting relations among the elements of CKM, which are fully experimentally measured values and obviously parametrization-independent,

$$
\begin{aligned}
& \frac{\left(\left|U_{13}\right|^{2}+\left|U_{33}\right|^{2}\right)\left(\left|U_{21}\right|^{2}+\left|U_{22}\right|^{2}\right)}{\left|U_{21}\right|\left|U_{22}\right|\left(\left|U_{23}\right|^{2}-1\right)} \approx \frac{\left(\left|U_{11}\right|^{2}+\left|U_{12}\right|^{2}\right)\left(\left|U_{23}\right|^{2}+\left|U_{33}\right|^{2}\right)}{\left|U_{11}\right|\left|U_{12}\right|\left(\left|U_{13}\right|^{2}-1\right)} \\
& \frac{\left(\left|U_{12}\right|^{2}+\left|U_{13}\right|^{2}\right)\left(\left|U_{21}\right|^{2}+\left|U_{31}\right|^{2}\right)}{\left|U_{11}\right|\left(\left|U_{11}\right|^{2}-1\right)\left|U_{12}\right|\left|U_{21}\right|} \approx \frac{\left(\left|U_{13}\right|^{2}+\left|U_{23}\right|^{2}\right)\left(\left|U_{31}\right|^{2}+\left|U_{32}\right|^{2}\right)}{\left|U_{23}\right|\left|U_{32}\right|\left|U_{33}\right|\left(\left|U_{33}\right|^{2}-1\right)} \\
& \frac{\left(\left|U_{13}\right|^{2}+\left|U_{33}\right|^{2}\right)\left(\left|U_{21}\right|^{2}+\left|U_{22}\right|^{2}\right)}{\left|U_{21}\right|\left|U_{23}\right|\left(\left|U_{23}\right|^{2}-1\right)\left|U_{33}\right|} \approx \frac{\left(\left|U_{11}\right|^{2}+\left|U_{13}\right|^{2}\right)\left(\left|U_{22}\right|^{2}+\left|U_{32}\right|^{2}\right)}{\left|U_{11}\right|\left|U_{12}\right|\left(\left|U_{12}\right|^{2}-1\right)\left|U_{32}\right|} \\
& \frac{\left(\left|U_{12}\right|^{2}+\left|U_{22}\right|^{2}\right)\left(\left|U_{31}\right|^{2}+\left|U_{33}\right|^{2}\right)}{\left|U_{12}\right|\left|U_{22}\right|\left(\left|U_{32}\right|^{2}-1\right)} \approx \frac{\left(\left|U_{11}\right|^{2}+\left|U_{21}\right|^{2}\right)\left(\left|U_{32}\right|^{2}+\left|U_{33}\right|^{2}\right)}{\left|U_{11}\right|\left|U_{21}\right|\left(\left|U_{31}\right|^{2}-1\right)} \\
& \frac{\left(\left|U_{12}\right|^{2}+\left|U_{22}\right|^{2}\right)\left(\left|U_{31}\right|^{2}+\left|U_{33}\right|^{2}\right)}{\left|U_{12}\right|\left|U_{32}\right|\left(\left|U_{32}\right|^{2}-1\right)\left|U_{33}\right|} \approx \frac{\left(\left|U_{11}\right|^{2}+\left|U_{31}\right|^{2}\right)\left(\left|U_{22}\right|^{2}+\left|U_{23}\right|^{2}\right)}{\left|U_{11}\right|\left|U_{21}\right|\left(\left|U_{21}\right|^{2}-1\right)\left|U_{23}\right|} .
\end{aligned}
$$

Because the CKM is a unitary matrix the following relations should hold

$$
\begin{aligned}
& \left|U_{11}\right|^{2}+\left|U_{12}\right|^{2}+\left|U_{13}\right|^{2}=1,\left|U_{21}\right|^{2}+\left|U_{22}\right|^{2}+\left|U_{23}\right|^{2}=1,\left|U_{31}\right|^{2}+\left|U_{32}\right|^{2}+\left|U_{33}\right|^{2}=1 \\
& \left|U_{11}\right|^{2}+\left|U_{21}\right|^{2}+\left|U_{31}\right|^{2}=1,\left|U_{12}\right|^{2}+\left|U_{22}\right|^{2}+\left|U_{32}\right|^{2}=1,\left|U_{13}\right|^{2}+\left|U_{23}\right|^{2}+\left|U_{33}\right|^{2}=1
\end{aligned}
$$

then Eq.(7) can be simplified to forms of

$$
\begin{aligned}
\frac{\left|U_{21}\right|\left|U_{22}\right|}{1-\left|U_{23}\right|^{2}} & \approx \frac{\left|U_{11}\right|\left|U_{12}\right|}{\left|U_{23}\right|^{2}+\left|U_{33}\right|^{2}} \\
\frac{\left|U_{11}\right|\left|U_{12}\right|\left|U_{21}\right|}{1-\left|U_{11}\right|^{2}} & \approx \frac{\left|U_{23}\right|\left|U_{32}\right|\left|U_{33}\right|}{1-\left|U_{33}\right|^{2}} \\
\frac{\left|U_{21}\right|\left|U_{23}\right|\left|U_{33}\right|}{1-\left|U_{23}\right|^{2}} & \approx \frac{\left|U_{11}\right|\left|U_{12}\right|\left|U_{32}\right|}{\left|U_{22}\right|^{2}+\left|U_{32}\right|^{2}} \\
\frac{\left|U_{12}\right|\left|U_{22}\right|}{1-\left|U_{32}\right|^{2}} & \approx \frac{\left|U_{11}\right|\left|U_{21}\right|}{\left|U_{11}\right|^{2}+\left|U_{21}\right|^{2}} \\
\frac{\left|U_{12}\right|\left|U_{32}\right|\left|U_{33}\right|}{\left|U_{12}\right|^{2}+\left|U_{22}\right|^{2}} & \approx \frac{\left|U_{11}\right|\left|U_{21}\right|\left|U_{23}\right|}{1-\left|U_{21}\right|^{2}} .
\end{aligned}
$$

Since the denominators of all quantities on both sides of Eq.(8) are small numbers and according to the general rule for numerical computations, handling such large fractions cannot guarantee higher accuracy, thus we take their reciprocals to build Eq.(10). 


\section{The corresponding relations in PMNS}

Since the CP-phase in PMNS is not well determined so far, in Ref. 114 the authors tried to fix $\delta_{c}^{\prime}$ in the corresponding $\mathrm{P}_{c}$ parametrization and then used it to determine $\delta_{n}^{\prime}$ in other parametrizations. By the values listed in Ref.[14] we note that relations similar to those in Eq.(7) also exist. Thus we can write out the relations in Eq.(10), even though the new relations where we replace $\delta_{n}$ in Eq.(7) by $\delta_{n}^{\prime}$, only roughly hold. Using the central value of $\vartheta_{a 1}, \vartheta_{a 2}$ and $\vartheta_{a 3}$ in Eq.(6) with $\delta_{a}^{\prime}=0^{\circ}$ we calculate the value in Eq.(10) and present the results in Tab. III. It is noted that those relations hold up to over $90 \%$ accuracy in this case.

To solve the solar neutrino missing problem, it is suggested that different flavor-neutrinos should also mix to compose physical states (mass eigenstates). Later people learned that the mixing patterns for neutrino may possess higher symmetries than for quarks and among many other symmetric configurations, a favorable form named as the bimaximal mixing pattern[17 19] is proposed

$$
\left|V_{T B}\right|=\left(\begin{array}{ccc}
\frac{1}{\sqrt{2}} & \frac{1}{\sqrt{2}} & 0 \\
\frac{1}{2} & \frac{1}{2} & \frac{1}{\sqrt{2}} \\
\frac{1}{2} & \frac{1}{2} & \frac{1}{\sqrt{2}}
\end{array}\right)
$$

which manifests a high symmetry among the three flavors. Let us investigate possible relations among those elements. Substituting those elements $\left|V_{T B}\right|$ into Eq.(10), one can find that the equalities given above are exact, and it is a very interesting issue. We will explore the exact solutions and their implications in our future work.

\section{NUMERICAL CHECK}

To confirm the validity of the relations obtained in last section, we should check them numerically. Using the central values of the measured quantities: $\left|U_{11}\right|=0.97427,\left|U_{12}\right|=$ $0.22535,\left|U_{13}\right|=0.00352,\left|U_{21}\right|=0.2252,\left|U_{22}\right|=0.97344,\left|U_{23}\right|=0.0412,\left|U_{31}\right|=0.00867$,

$\left|U_{32}\right|=0.0404$ and $\left|U_{33}\right|=0.999145[20]$ which are the best fit from experiments, one can calculate all the concerned quantities in Eq.(10) (i.e. the combinations of the matrix elements). The results are shown in the table III. It is not a surprise to find that the first four equations hold with very high accuracy whereas the last one declines from rigorous equality by a few percents. Even though this deviation is not large, we still feel puzzled about the reason, it might be caused by experimental errors for measuring the involved quantities or there exists a more profound cause. Indeed, in the last section, we will return to make a short discussion on this issue. Let us make more numerical computations. If one uses equal signs to replace " $\approx$ " in Eq.(10) which involve seven elements of $\left|U_{j k}\right|$, and takes $\left|U_{12}\right|=0.22535,\left|U_{23}\right|=$ 0.0412 and $\left|U_{33}\right|=0.999145$ as inputs, by solving the four equations in Eq.(10), he would obtain, $\left|U_{11}\right|=0.974066,\left|U_{21}\right|=0.225292,\left|U_{22}\right|=0.972675$ and $\left|U_{32}\right|=0.0401085$ which are very close to the central values of the data as shown above.

Furthermore, as we argued above, those equalities are scheme-independent, and there are only four parameters for a unitary $3 \times 3$ matrix, thus by solving the equations we may expect 
TABLE II: The values in Eq.(10) for PMNS.

\begin{tabular}{c|c|c|c}
\hline Equality No. & Left side & Right side & Relative deviation \\
\hline 1 & 0.49429 & 0.461269 & $6.68054 \%$ \\
2 & 0.715365 & 0.692966 & $3.1311 \%$ \\
3 & 0.392148 & 0.375742 & $4.18372 \%$ \\
4 & 0.49799 & 0.449634 & $9.71014 \%$ \\
5 & 0.376304 & 0.35314 & $6.15571 \%$ \\
\hline
\end{tabular}

TABLE III: The values in Eq.(10) for CKM.

\begin{tabular}{c|c|c|c}
\hline Equality No. & Left side & Right side & Relative deviation \\
\hline 1 & 0.219591 & 0.219554 & $0.0168495 \%$ \\
2 & 0.973327 & 0.972964 & $0.0372948 \%$ \\
3 & 0.00928607 & 0.00934442 & $0.628361 \%$ \\
4 & 0.219723 & 0.219424 & $0.13608 \%$ \\
5 & 0.00911122 & 0.00952312 & $4.5208 \%$ \\
\hline
\end{tabular}

to get all $\left|U_{j k}\right|$ up to a universal constant. Let us try and see if we can reach this goal.

As we guessed, the aforementioned equalities can determine all the matrix elements up to a universal constant which could be the scheme-independent Jarlskog invariant. Since the Jarlskog invariant should be written in terms of the scheme-dependent parameters (mixing angles $\theta_{n j}$ and CP phase $\delta_{n}$ ), it is not convenient to use for our computations, instead, we arbitrarily choose a few $\left|U_{j k}\right|$ values which are experimentally measured, thus scheme-independent as inputs. By manipulating the equations in Eq.(9), we have two relations $U_{21}^{2}+U_{22}^{2}+U_{23}^{2}=1$ and $U_{11}^{2}+U_{21}^{2}-U_{32}^{2}-U_{33}^{2}=0$. Using them and the first three equalities in Eq.(10) we obtain $\left|U_{11}\right|=0.974278,\left|U_{21}\right|=0.225159,\left|U_{22}\right|=0.973451\left|U_{32}\right|=0.0401402$ and $\left|U_{33}\right|=0.999151$ by setting $\left|U_{12}\right|=0.22535$ and $\left|U_{23}\right|=0.0412$. The results are satisfactorily consistent with the measured data. While further including the fourth equalities in Eq.(10) and only inputting one matrix element, say $\left|U_{12}\right|$, we find that directly solving the equation group becomes very complicated and our computer program refuses to give a numerical result. Instead, as the iterative method is employed, with $\left|U_{12}\right|=0.22535$ as input, we immediately obtain $\left|U_{11}\right|=0.974278,\left|U_{21}\right|=0.225164,\left|U_{22}\right|=0.973473\left|U_{32}\right|=0.039599,\left|U_{32}\right|=0.0406445$ and $\left|U_{33}\right|=0.999174$. Comparing with the data, the predictions are even better than expectation.

The four equations seem to be independent, so one may wish to fix all the three mixing angles and one CP-phase simultaneously. However, it is obviously impossible because there are no any numbers in those equations except 1 . As all the $\left|U_{j k}\right|$ 's are irrational numbers, so one cannot expect to gain them by solving such equations. Instead, we may try to find some valuable information about the $\mathrm{CP}$ phase via the $\chi^{2}$ method [21, 22]. For example in $\mathrm{P}_{a}$ parametrization the three CKM mixing angles are $\theta_{a 1}=13.023^{\circ}, \theta_{a 2}=2.360^{\circ}, \theta_{a 3}=0.201^{\circ}$, 
we would calculate $\chi^{2}$ which is defined as

$$
\begin{aligned}
\chi^{2}= & \left.\frac{\left|U_{21}\right|\left|U_{22}\right|}{1-\left|U_{23}\right|^{2}}-\frac{\left|U_{11}\right|\left|U_{12}\right|}{\left|U_{23}\right|^{2}+\left|U_{33}\right|^{2}}\right)^{2}+\left(\frac{\left|U_{11}\right|\left|U_{12}\right|\left|U_{21}\right|}{1-\left|U_{11}\right|^{2}}-\frac{\left|U_{23}\right|\left|U_{32}\right|\left|U_{33}\right|}{1-\left|U_{33}\right|^{2}}\right)^{2}+ \\
& \left(\frac{\left|U_{21}\right|\left|U_{23}\right|\left|U_{33}\right|}{1-\left|U_{23}\right|^{2}}-\frac{\left|U_{11}\right|\left|U_{12}\right|\left|U_{32}\right|}{\left|U_{22}\right|^{2}+\left|U_{32}\right|^{2}}\right)^{2}+\left(\frac{\left|U_{12}\right|\left|U_{22}\right|}{1-\left|U_{23}\right|^{2}}-\frac{\left|U_{11}\right|\left|U_{21}\right|}{\left|U_{11}\right|^{2}+\left|U_{21}\right|^{2}}\right)^{2}
\end{aligned}
$$

The result is depicted in Fig. 1(a). One can notice that there exist two minima of $\chi_{\min }^{2}$ at $\delta_{a} \approx 68.75^{\circ}$ which fall in the tolerance range of the data $\left(69.10_{-3.85}^{+2.02}\right)^{\circ}$ and its complementary position at $291.06^{\circ}$.

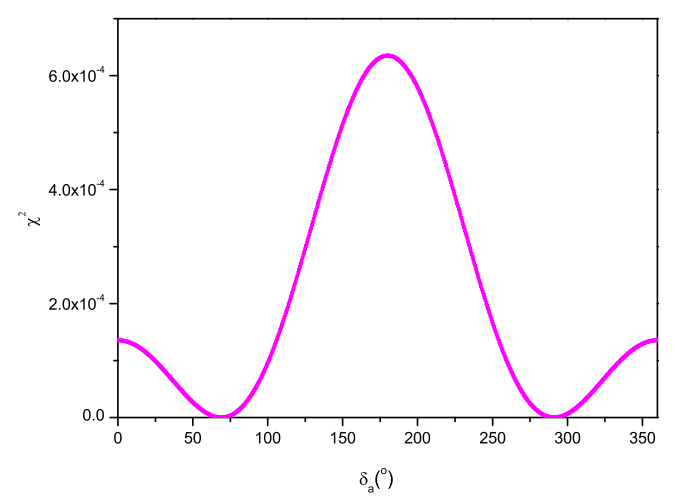

(a)

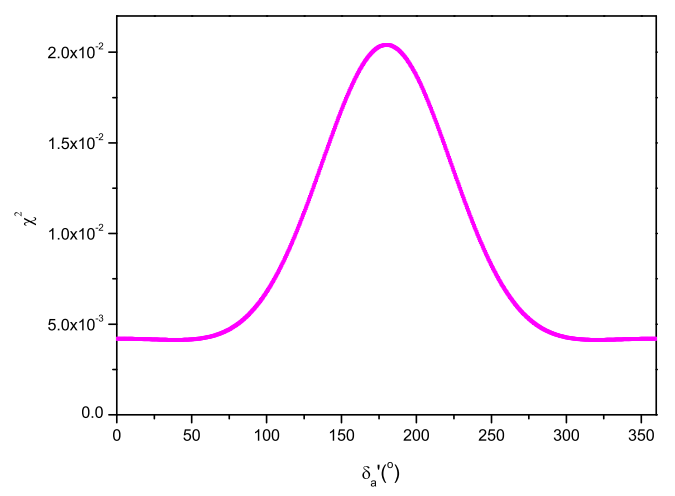

(b)

FIG. 1: The dependence of $\chi^{2}$ which is defined in Eq.(12) on the CP phase $\delta$ (a, for CKM ); and $\delta^{\prime}(\mathrm{b}$, for PMNS) .

Then we extend the same calculation to the PMNS case. With the mixing angles $\vartheta_{a 1}=33.65^{\circ}, \vartheta_{a 2}=38.41^{\circ}$ and $\vartheta_{a 3}=8.93^{\circ}$ in $\mathrm{P}_{a}$ parametrization which are experimentally measured with certain errors, we compute corresponding $\chi^{2}$ which depends on $\delta_{a}^{\prime}$ and the result is presented in Fig. 1(b). It is also noted, the value of $\chi^{2}$ does not vary much within a relatively wide range $0^{\circ}$ to $59^{\circ}$ centered at $39^{\circ}$. Therefore, it is hard to firmly fix the CP phase at the lepton sector with this $\chi^{2}$ method, and one needs to wait for the more accurate long-baseline neutrino experiments in the future to determine its value. Our estimate merely indicates its possible range.

\section{SUMMARY AND DISCUSSIONS}

As is well known, the CKM and PMNS matrices emerge due to the mismatch between the mass eigenstates and flavor eigenstates in the quark and lepton sectors respectively. The 
masses of different generations are not the same and compose the hierarchy problem. That is one of the unknown parts in particle physics. There must be sort of association between the mass hierarchy and mixing matrix. In a recent paper Xing [23] reviews the situation and extends the discussion from the quark sector to the lepton sector. Merlo also discusses the neutrino masses and mixing from continuous symmetries [24]. Especially, the mass hierarchy problem of neutrinos i.e. it is either normal or inverted, is seriously considered and the JUNO experiment which will operate in China in a few years, will determine it.

There are still more mysteries for the mixing, for example, the complementarity relations between elements of CKM and PMNS matrices and the self-complementarities among the matrix elements of PMNS [9 15]. It was studied that the obvious symmetry in PMNS can be traced to the possible symmetric textures for lepton masses [25, 26], thus whether a fundamental symmetry really exists and what it is, is still an unsolved problem in our theoretical prospect. Therefore, we are tempted to investigate the CKM and PMNS matrices from a new angle and hope to gain a better understanding of the possible underlying theories.

We note several relations among the CKM matrix parameters of the nine parametrization schemes i.e. $\sin \delta_{a} \approx \sin \delta_{e}, \sin \delta_{b} \approx \sin \delta_{c}, \sin \delta_{d} \approx \sin \delta_{e}, \sin \delta_{f} \approx \sin \delta_{h}, \sin \delta_{h} \approx \sin \delta_{i}$, by which we establish several scheme-independent equalities. Those relations may imply existence of something which is independent of any concrete parametrization.

The Jarlskog invariant is a physically measurable quantity, so must be the same for all the schemes which include the sine values of the CP phase $\delta_{n}$.

By the observation, it was suggested that the mixing matrices originate from a higher symmetry [27], and the nine parametrization schemes are merely various representations of the unique physical mixing matrix, therefore the relations among the sine values of the CP phases of the nine schemes actually reveal the inherent essence of the symmetry. We check these equalities numerically and find the first four hold with very high precision. Moreover, if the underlying symmetry really exists, the elements of the CKM matrix which seem to be in an anarchy state at first glimpse, might be well arranged according to a certain rule. Thus we would see whether the CKM matrix elements can be completely determined from those equalities up to a universal constant, which is the Jarlskog invariant.

On the other aspect, most of the symmetries in the nature are somewhat broken. Mostly, the breaking might not be too large, so people still can trace back to the original symmetry. For example, we proposed that breaking of the complementarity and self-complementarity between CKM and PMNS matrices is due to the involvement of a sterile neutrino[28]. Here we can observe that the relations of the CKM matrix elements hold with high precision, but an approximation degree is also noted as $0.02 \% \sim 0.63 \%$. Especially, the fifth relation listed in Eq.(10) deviates from equality by $4.52 \%$.

It is naturally conjectured that the relations obtained for the CKM matrix can also be applied to the PMNS matrix. Because the PMNS matrix elements are not well measured as that of the CKM matrix, we can only expect that the relations among the PMNS matrix elements only approximately hold. For the lepton case we can also estimate $\delta_{a}^{\prime}$ in terms of the $\chi^{2}$ method, the resultant $\delta_{a}^{\prime} \approx 39^{\circ}$. But as shown in the Fig. 11(b), the value $\chi^{2}$ does not vary much from $0^{\circ}$ to $59^{\circ}$. Therefore, we cannot firmly determine the $\mathrm{CP}$ phase for the 
lepton sector as much as we do for the quark sector from the relations yet.

Our observation on the relations indicates that a symmetry at higher scale does exist and after running down to the low energy scale its essential characters remain. Therefore, even though it is slightly broken, we still can trace back to the original symmetry. Further studies

on the higher symmetry is definitely needed to eventually understand these relations.

\section{Acknowledgement}

This work is supported by the National Natural Science Foundation of China (NNSFC) under the contract No. 11375128 and 11135009.

\section{Appendix A}

[1] M. Veltman, facts and mysteries in elementary particle physics, World Scientific Publishing Co. Pte. Ltd. (2003).

[2] N. Cabibbo, Phys. Rev. Lett. 10, 531 (1963).

[3] M. Kobayashi and T. Maskawa, Prog. Theor. Phys. 49, 652 (1973).

[4] H. Fritzsch, Nucl. Phys. B 155, 189 (1979).

[5] L. F. Li, Phys. Lett. B 84, 461 (1979).

[6] H. Fritzsch and Z. Z. Xing, Phys. Lett. B 413, 396 (1997) hep-ph/9707215.

[7] B. Pontecorvo, Sov. Phys. JETP 26, 984 (1968) [Zh. Eksp. Teor. Fiz. 53, 1717 (1967)].

[8] Z. Maki, M. Nakagawa and S. Sakata, Prog. Theor. Phys. 28, 870 (1962).

[9] H. Minakata and A. Y. .Smirnov, Phys. Rev. D 70, 073009 (2004) hep-ph/0405088.

[10] M. Raidal, Phys. Rev. Lett. 93, 161801 (2004) hep-ph/0404046.

[11] G. Altarelli, F. Feruglio and L. Merlo, JHEP 0905, 020 (2009) arXiv:0903.1940 [hep-ph]]; G. Altarelli and D. Meloni, J. Phys. G 36, 085005 (2009) [arXiv:0905.0620 [hep-ph]]; R. de Adelhart Toorop, F. Bazzocchi and L. Merlo, JHEP 1008, 001 (2010) arXiv:1003.4502 [hep-ph]]; G. Altarelli, F. Feruglio, L. Merlo and E. Stamou, JHEP 1208, 021 (2012) arXiv:1205.4670 [hep-ph]].

[12] Y. -j. Zheng and B. -Q. Ma, Eur. Phys. J. Plus 127, 7 (2012) arXiv:1106.4040 [hep-ph]]; X. Zhang and B. -Q. Ma, Phys. Rev. D 86, 093002 (2012) arXiv:1206.0519 [hep-ph]]; H. Qu and B. -Q. Ma, Phys. Rev. D 88, 037301 (2013) arXiv:1305.4916 [hep-ph]].

[13] X. Zhang, Y. -j. Zheng and B. -Q. Ma, Phys. Rev. D 85, 097301 (2012) arXiv:1203.1563 [hep-ph]].

[14] Y. Zhang, X. Zhang and B. -Q. Ma, Phys. Rev. D 86, 093019 (2012) [arXiv:1211.3198[hep-ph]].

[15] N. Haba, K. Kaneta and R. Takahashi, Europhys. Lett. 101, 11001 (2013) arXiv:1209.1522 [hep-ph]]. 
[16] C. Jarlskog, Phys. Rev. Lett. 55, 1039 (1985); D. d. Wu, Phys. Rev. D 33, 860 (1986); O. W. Greenberg, Phys. Rev. D 32, 1841 (1985).

[17] F. Vissani, hep-ph/9708483.

[18] V. D. Barger, S. Pakvasa, T. J. Weiler and K. Whisnant, Phys. Lett. B 437, 107 (1998) hep-ph/9806387.

[19] A. J. Baltz, A. S. Goldhaber and M. Goldhaber, Phys. Rev. Lett. 81, 5730 (1998) hep-ph/9806540.

[20] J. Beringer et al. [Particle Data Group Collaboration], Phys. Rev. D 86, 010001 (2012).

[21] C. -W. Chiang, M. Gronau, J. L. Rosner and D. A. Suprun, Phys. Rev. D 70, 034020 (2004) hep-ph/0404073.

[22] H. -W. Ke, J. Tang, X. -Q. Hao and X. -Q. Li, Phys. Rev. D 76, 074035 (2007) arXiv:0706.2074 [hep-ph]].

[23] Z. z. Xing, arXiv:1411.2713 [hep-ph].

[24] L. Merlo, arXiv:1411.2766 [hep-ph].

[25] B. Wang, J. Tang and X. Li, Phys.Rev. D88 073003 (2014).

[26] J. Y. Liu and S. Zhou, Phys. Rev. D 87, no. 9, 093010 (2013) arXiv:1304.2334 [hep-ph]].

[27] C. S. Lam, arXiv:1105.4622 [hep-ph]; C. S. Lam, Phys. Rev. D 83, 113002 (2011) arXiv:1104.0055 [hep-ph]].

[28] H. W. Ke, T. Liu and X. Q. Li, Phys. Rev. D 90, 053009 (2014) arXiv:1408.1315 [hep-ph]]. 